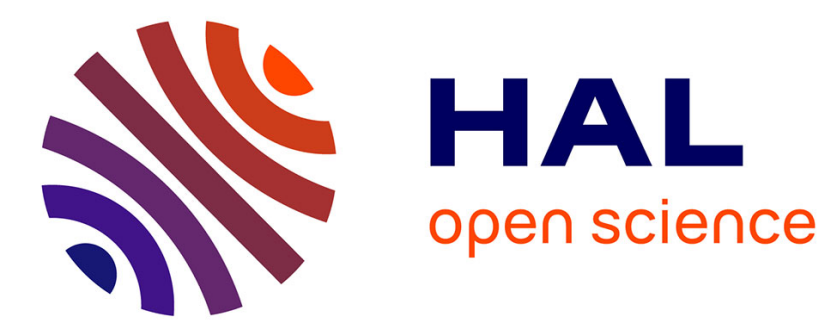

\title{
SPIN IN ANTINUCLEON PHYSICS
}

F. Bradamante

\section{To cite this version:}

F. Bradamante. SPIN IN ANTINUCLEON PHYSICS. Journal de Physique Colloques, 1990, 51 (C6), pp.C6-299-C6-316. 10.1051/jphyscol:1990624 . jpa-00230890

\section{HAL Id: jpa-00230890 https://hal.science/jpa-00230890}

Submitted on 1 Jan 1990

HAL is a multi-disciplinary open access archive for the deposit and dissemination of scientific research documents, whether they are published or not. The documents may come from teaching and research institutions in France or abroad, or from public or private research centers.
L'archive ouverte pluridisciplinaire HAL, est destinée au dépôt et à la diffusion de documents scientifiques de niveau recherche, publiés ou non, émanant des établissements d'enseignement et de recherche français ou étrangers, des laboratoires publics ou privés. 


\title{
SPIN IN ANTINUCLEON PHYSICS
}

\section{F. BRADAMANTE}

Dipartimento di Fisica dell'Universita, Trieste, Italy and Sezione di Trieste dell'INFN, Trieste, Italy

\begin{abstract}
Recent antiproton-proton data, mostly coming from the Low-Energy Antiproton Ring, LEAR, at CERN, are illustrated and their trend discussed. The data are compared with one-boson-exchange models to extract the global behaviour of annihilation. Some ideas for future measurements and developments are presented.

Les données antiproton-proton obtenues en grande majorité à l'Anneau de stockage d'antiprotons à basse énergie du CERN, LEAR, sont illustrées et discutées. Ces données sont comparées aux prévisions des modèles potentiels avec échange de bosons, dans le but d'obtenir les caractéristiques globales des processus d'annihilation. Enfin, des idées de mesures futures et de développements sont présentées.
\end{abstract}

\section{INTRODUCTION}

Since the commissioning of the Low-Energy Antiproton Ring (LEAR) [1] at CERN, in 1983, precise $N \bar{N}$ data have become available. Although in the past several laboratories contributed to low-energy antiproton physics, and to quote a recent result I should like to recall the work done in Brookhaven and at KEK in the early 80 's, by now the quality of the LEAR beam is so superior that CERN is the only laboratory where this physics is pursued. The momentum of the extracted beam can range from 70 to $2000 \mathrm{MeV} / c$. Apart from the very low momenta, the beam intensity is routinely $2 \times 10^{6} / \mathrm{s}$, with horizontal and vertical emittances of 2 and $5 \pi \cdot \mathrm{mm} \cdot \mathrm{mrad}$, respectively. The momentum spread $\Delta p / p$ is less than $10^{-3}$. The beam is extracted from LEAR continuously, and delivered to up to three experiments, typically over one hour, while about ten minutes are necessary to transfer a new spill from the Antiproton Accumulator (ACOL), to cool and accelerate (or decelerate) it to the desired extraction value.

After some general comments on the motivations for $N \bar{N}$ scattering, and a brief review of the results of the experiments performed before the machine shutdowa for the construction of the new CERN Antiproton Collector (ACOL), 
I will discuss the new data, mostly on polarization. In this written version of my talk I will not mention the $\bar{p} p \rightarrow \bar{\Lambda} \Lambda$ results, which are the object of a separate contribution [2]. I will then illustrate the present theoretical understanding of the $N \bar{N}$ data, in the framework of some well-known one-boson-exchange (OBE) potential models. In the last section I will describe some present ideas for future measurements.

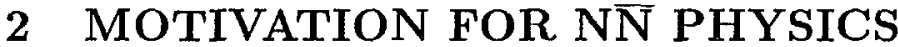

The study of the $N \bar{N}$ interaction at low energy is complementary to the study of the NN interaction, and one cannot say that one understands the latter without being able to explain the former. Although the ultimate goal is the understanding of hadron dynamics at the microscopic level, the comparison between data and theory is usually done by using potential models.

The basic ingredients of the $N \bar{N}$ interaction are

- meson exchanges at large distances $(r \gtrsim 0.8 \mathrm{fm})$. These are assumed to be the same as determined in the NN interaction, and the sign of each contribution is obtained from the G-parity rule [3]:

$$
V_{\mathrm{N} \overline{\mathrm{N}}}=\sum_{\pi p w \sigma \ldots}(-1)^{G} V_{\mathrm{NN}}(\mathrm{OBE}) ;
$$

- annihilation at short range, usually parametrized as an optical potential, eventually state and energy dependent.

It is well-known that the $G$-parity rule changes dramatically the overall $N \bar{N}$ meson-exchange potential as compared with the NN case. As an example, 1 will just recall the special role of $\omega$-exchange, which gives rise to a repulsive force in $N N$ interactions, and to a strong attraction in the $N \vec{N}$ case. In this sense the study of the long-range part of the $\mathrm{N} \overline{\mathrm{N}}$ interaction is clearly complementary to that of the NN channels, and could provide new constraints on the OBE potential models. In addition to this, $N \bar{N}$ physics has some unique features, i.e.

- the quarks' degrees of freedom play a much more prominent role than in NN (annihilation in N $\bar{N}$ as compared with core repulsion in the latter case);

- annihilation itself is a challenge for any theory of hadron constituents;

- both $N \bar{N}$ scattering and annihilation are expected to give privileged access to exotic states in the s-channel (Fig. 1).

Before LEAR came into operation the quality of the secondary $\bar{p}$ beams was such that the study of the $N \bar{N}$ interaction could not be adequately pursued. In particular, spin observables, the necessary tool to unfold the dynamics of a four-fermion system, were essentially unmeasured. As I will show in 
Figure 1: Quark diagrams for exotic states in $N \bar{N}$ interactions (baryonium and gluonium).

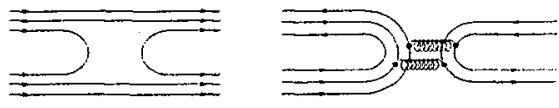

Figure 2: Data on $\bar{p} p$ total cross-section (full dots) and on p p annihilation cross-section (open triangles) versus $\overline{\mathrm{p}}$ momentum. The curves are fits to the data [6], [7].

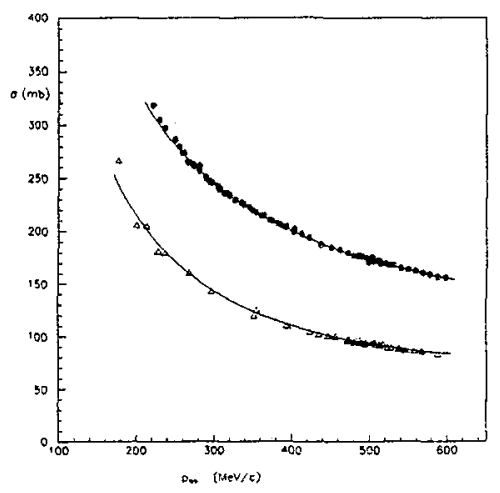

Section 4, the situation has changed, and precise experimental data are now available.

\section{CROSS-SECTION AND SCATTERING RE- SULTS}

Although almost all of these data are published, I thought it useful to review them for completeness. Most of the results came from the experiments PS172 and PS1731,2.

\subsection{Cross-section data}

Total $\bar{p}$ cross-section data [4] and annihilation cross-section data [5] are shown in Fig. 2. The trend of the data with momentum is very smooth, and no narrow peaks are seen, thus confirming the KEK [6] and BNL [7] results demonstrating the non-existence of a narrow 'baryonium' peak at $500 \mathrm{MeV} / \mathrm{c}$, the $\mathrm{S}(1936)$, previously seen in several experiments [8]. Furthermore, the cross-sections measured at BNL [9] in the $\bar{n} p$ channel (pure $I=1$ system) look very smooth between 100 and $500 \mathrm{MeV} / c$, and no narrow structures are visible.

\footnotetext{
${ }^{1}$ Amsterdarn-Geneva-Queen Mary College-Surrey-Trieste Collaboration, spokesman D.V. Bugg.

${ }^{2}$ Heidelberg MPI and University Collaboration, spokesman Th. Walcher.
} 


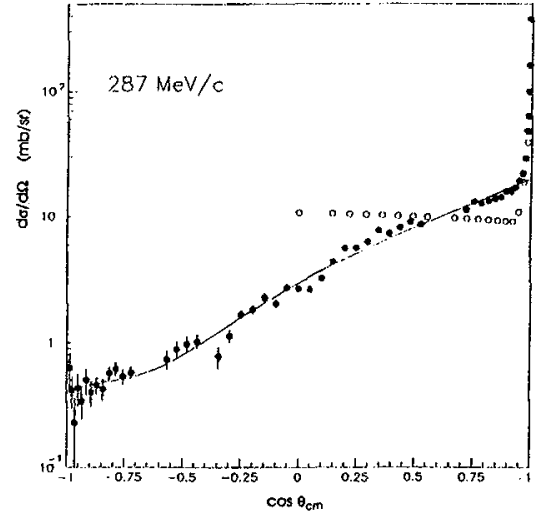

(a)

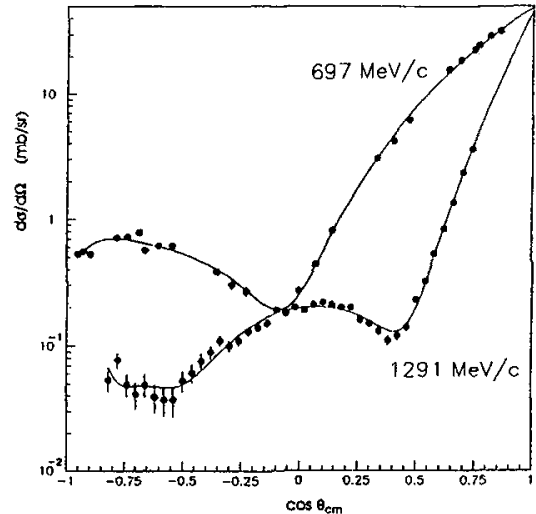

(b)

Figure 3: Differential elastic cross-section data for $\bar{p} p$ (a) at $287 \mathrm{MeV} / \mathrm{c}$ (also shown pp data at $275 \mathrm{MeV} / c$ ); (b) at $697 \mathrm{MeV} / c$ and at $1291 \mathrm{MeV} / c$. The curves are Legendre fits.

\subsection{Elastic $\bar{p} p$ scattering}

Differential cross-sections have been measured with good precision at low momenta $(181,287$, and $505 \mathrm{MeV} / \mathrm{c})$ by experiment PS173 [10], at intermediate momenta $(439,544$, and $697 \mathrm{MeV} / \mathrm{c})$ by experiment PS198 ${ }^{3}$, and at fifteen momenta between 530 and $1550 \mathrm{MeV} / \mathrm{c}$ by experiment PS172 [11]. The main feature of the $\bar{p} p$ differential cross-section is the strong p-wave enhancement, which manifests itself as the forward peak present even at very low energies. This can be seen in Fig. 3a, where the $\bar{p} p$ and pp differential cross-sections at $\sim 50 \mathrm{MeV}$ kinetic energy are compared: the s-wave pp scatlering contrasts sharply with the two-orders-of-magnitude forward-backward cross-section difference in the $\bar{p} p$ case. This diffraction behaviour is even more pronounced at higher momenta. Fig. 3b shows the published result from PS198, a full angular distribution, measured at $697 \mathrm{MeV} / c$ [12], well in agreement with existing data [13], and the data at one of the fifteen momenta measured by PS172, where a second diffraction minimum is already visible; again, the agreement with existing data is good [14].

The $\rho$-parameter, the ratio of the real-to-imaginary part of the forward elastic amplitude, has also been measured at seven momenta from 181 to $590 \mathrm{MeV} / \mathrm{c}$ by experiment PS173 [15] and at five momenta from 233 to $1100 \mathrm{MeV} / \mathrm{c}$ by ex-

\footnotetext{
${ }^{3}$ Karlsruhe-IPN Lyon-PSI-Saclay Collaboration, spokesman R. Bertini.
} 
Figure 4: A compilation of $\rho$, the ratio of the real-to-imaginary part of the forward elastic $\bar{p} p$ amplitude (Ref. [16]).

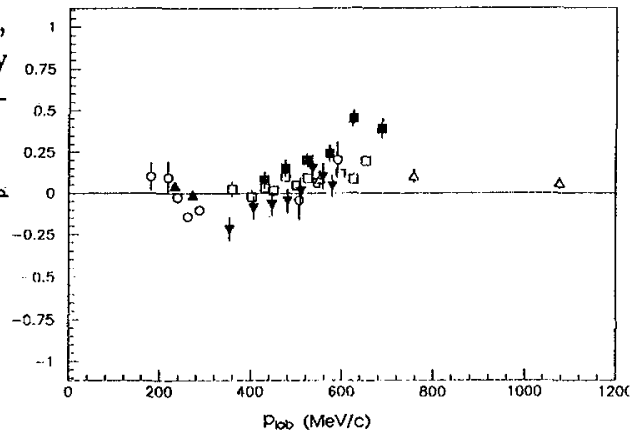

periment PS172 [16]. A compilation of these and previous data is shown in Fig. 4: apart from some small discrepancies among the data, $\rho$, very much like $\sigma_{\text {tot }}$, exhibits a very smooth dependence on momentum, and is rather small, decreasing from $\sim 0.1$ at high momentum to $\sim 0$ at $\sim 200 \mathrm{MeV} / \mathrm{c}$, in agreement with a recent dispersion relation analysis [17].

\subsection{Charge-exchange $\overline{\mathbf{p}} \mathbf{p} \rightarrow \overline{\mathbf{n}} \mathbf{n}$}

In contrast with the pp case, the p p system is not a pure isospin state, and the amplitude for pp elastic scattering is given by the sum of the $I=0$ and $I=1$ amplitudes. To resolve the isospin structure of the interaction it is essential therefore to measure also the isospin orthogonal channel, i.e. the charge-exchange reaction $\overline{\mathrm{p} p} \rightarrow \overline{\mathrm{n}} \mathrm{n}$.

The charge-exchange reaction is expected to be a particularly sensitive channel to probe the antinucleon-nucleon force. On the one hand, the long-range part should be dominated by pion exchange, a 'classical' term in any OBE potential model. On the other hand, since its amplitude is given by the difference of the $I=0$ and $I=1$ amplitudes, it should provide a good test of isospin independence.

Experimentally, it is a much more difficult channel to measure than the elastic channel. Experiment PS173 has measured the differential cross-section at four momenta $(183,287,505$, and $590 \mathrm{MeV} / \mathrm{c})$, providing the first data below $300 \mathrm{MeV} / \mathrm{c}$ [18]. The agreement with existing data at similar momenta [19], [20] is poor, as can be seen in Fig. 5a, where the LEAR data are compared with data from KEK. Quite recently, a new experiment, PS199 ${ }^{4}$, has provided highstatistics measurements at $693 \mathrm{MeV} / \mathrm{c}$ [21], which are compared with the KEK data in Fig. 5b.

\footnotetext{
${ }^{4}$ Cagliari-Geneva-Saclay-Trieste-Turin Collaboration, spokesman F. Bradamante.
} 


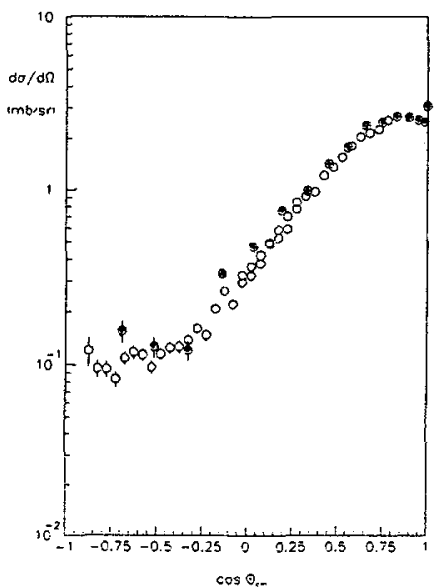

(a)

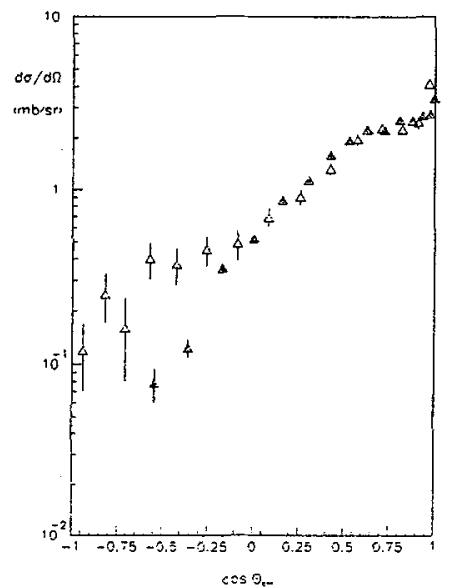

(b)

Figure 5: $\bar{p} \mathrm{p} \rightarrow \overline{\mathrm{n}} \mathrm{n}$ differential cross-section data from KEK (full points) and from LEAR (open points) at $590 \mathrm{MeV} / c$ (a) and $690 \mathrm{MeV} / c$ (b).

\subsection{Comparison with theory}

All the cross-section data seen so far can be described surprisingly well by a large variety of potential models [22]. In particular, only $\pi$-exchange and a suitably parametrized annihilation potential, with an annihilation radius of $\sim$ $0.8 \mathrm{fm}$, already give a satisfactory description of the data. For instance, a Woods-Saxon type annihilation potential, with

$$
\operatorname{Re} V_{\mathrm{ann}}=\frac{-c_{0}}{1+\exp \left[\left(r-r_{0}\right) / a_{0}\right]}, \quad \operatorname{Im} V_{\mathrm{ann}}=\frac{-c_{1}}{1+\exp \left[\left(r-r_{1}\right) / a_{1}\right]},
$$

and no energy, spin, or isospin dependence, already gives very good fits with $c_{0}=c_{1}=500 \mathrm{MeV}, r_{0}=r_{1}=0.74 \mathrm{fm}$, and $a_{0}=a_{1}=0.2 \mathrm{fm}$ [23]. A recent detailed comparison of the cross-section data with potential models calculations can be found in Ref. [13].

\section{MEASUREMENTS OF SPIN OBSERVABLES}

The advent of LEAR allowed good measurements of analysing power to be performed in the elastic $\bar{p}$ p channel by both experiments PS172 and PS198. 
At present, experiment PS199 is measuring the analysing power in the chargeexchange channel.

\subsection{The elastic $\overline{\mathbf{p}} \mathbf{p} \rightarrow \overline{\mathbf{p}} \mathbf{p}$ channel}

The analysing power $A_{0 n}$ has been measured over most of the angular range by experiment PS172 [11] at fifteen momenta, ranging from 530 to $1550 \mathrm{MeV} / \mathrm{c}$, The measurements are shown in Fig. 6 and compared with the predictions of the Dover-Richard model [24] and of the Paris model [25]. Experiment PS198 has measured $A_{0 n}$ over the entire angular range at 497, 523, and $697 \mathrm{MeV} / \mathrm{c}$. The data at $697 \mathrm{MeV} / \mathrm{c}$ are already published [12]: new results [26] at the two lowest momenta are shown in Fig. 7, together with previous data from experiment PS172 and predictions from the Paris, the Dover-Richard, and the Nijmegen [27] models.

The analysing power exhibits a lot of structure with angle and momentum, which is rather poorly reproduced by the potential models. There is good agreement between the new data, which anyway represent either a vast improvement over previous measurements [28], or cover a region where data did not exist (momenta smaller than $910 \mathrm{MeV} / c$ ).

By analysing the polarization of the scattered proton with a carbon polarimeter, experiment PS172 could obtain some $D_{0 n 0 n}$ data in the backward hemisphere and in the higher momentum range (from 1000 to $1550 \mathrm{MeV} / \mathrm{c}$ ). Some of these data [29] are shown in Fig. 8: although the error bars are large, the result is interesting because it suggests either zero or negative values for $D_{0 n 0 n}$ (except at $1291 \mathrm{MeV} / \mathrm{c}$ ), while the potential models would like this parameter to be close to 1 . Data on $D_{0 n 0 n}$. have been collected also by experiment PS198 at $700 \mathrm{MeV} / \mathrm{c}$, but the analysis is still in progress.

\subsection{The two-pion annihilation channel}

Experiment PS172 has also measured [30], at eighteen momenta ranging from 467 to $1550 \mathrm{MeV} / \mathrm{c}$, the analysing power of the annihilation channels $\overline{\mathrm{p}} \mathrm{p} \rightarrow$ $\pi^{+} \pi^{-}$and $\bar{p} \mathrm{p} \rightarrow \mathrm{K}^{+} \mathrm{K}^{-}$. Spectacular spin effects have been found, as can be seen in the compilation of Fig. 9. First attempts to describe the data in terms of the Paris potential models are not very successful [31]. In the past a phase-shift analysis of similar data in the momentum range 1000 to $2000 \mathrm{MeV} / \mathrm{c}$ has led to the identification of several high spin resonances [32].

\subsection{The charge-exchange channel}

The analysing power of the charge-exchange $\bar{p} p \rightarrow \bar{n} n$ reaction has been measured for the first time at low momentum (a previous measurement [33] exists at $8 \mathrm{GeV} / \mathrm{c}$ ) by experiment PS199. Data lave been collected at eight different incident $\overline{\mathrm{p}}$ momenta, ranging from 600 to $1300 \mathrm{MeV} / \mathrm{c}$. First results [21] at 656 


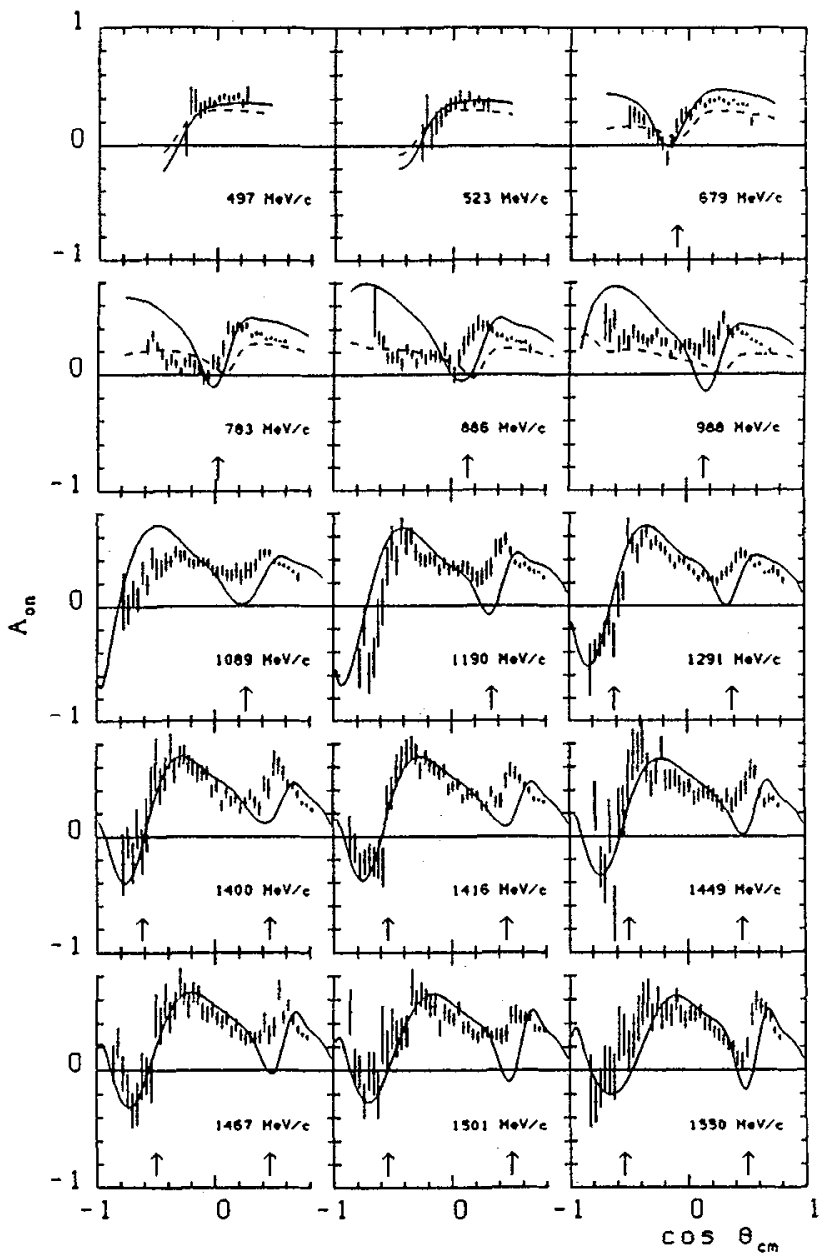

Figure 6: Analysing power for $\bar{p}$ p elastic scattering at many momenta (Ref. [11]). The dashed and full curves are predictions from the model of Refs. [24] and [25], respectively. 


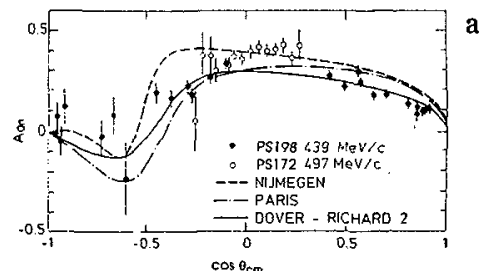

a)

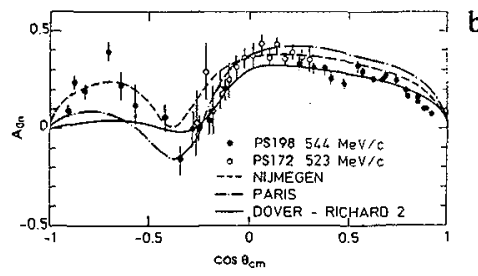

Figure 7: Analysing power for pp elastic scattering at two momenta. The curves are predictions from potential models, Dover-Richard [24], Paris [25], Nijmegen [26].
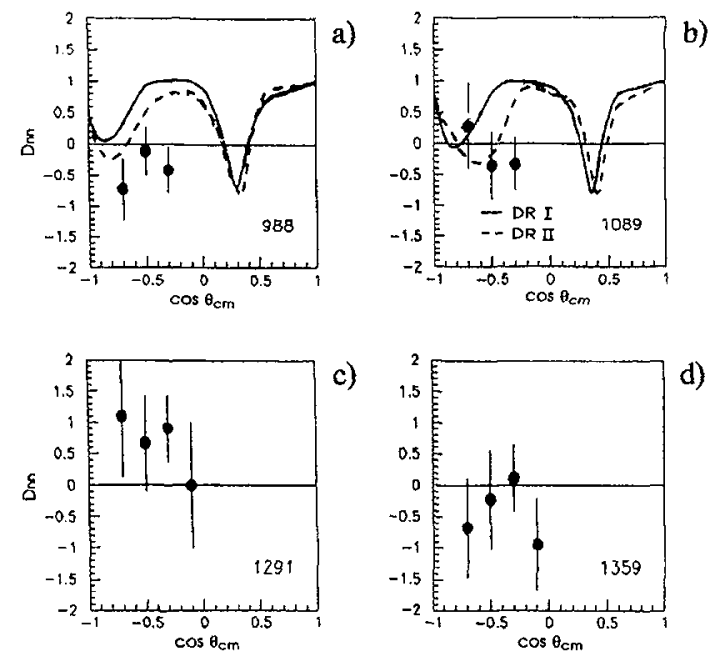

Figure 8: $D_{n n}$ results for $\overline{\mathrm{p}}$ elastic scattering at $988,1089,1291$, and $1359 \mathrm{MeV} / \mathrm{c}$. Theoretical predictions are from the models of Ref. [24]: Dover-Richard I (full curve) and Dover-Richard II (dashed curve). 


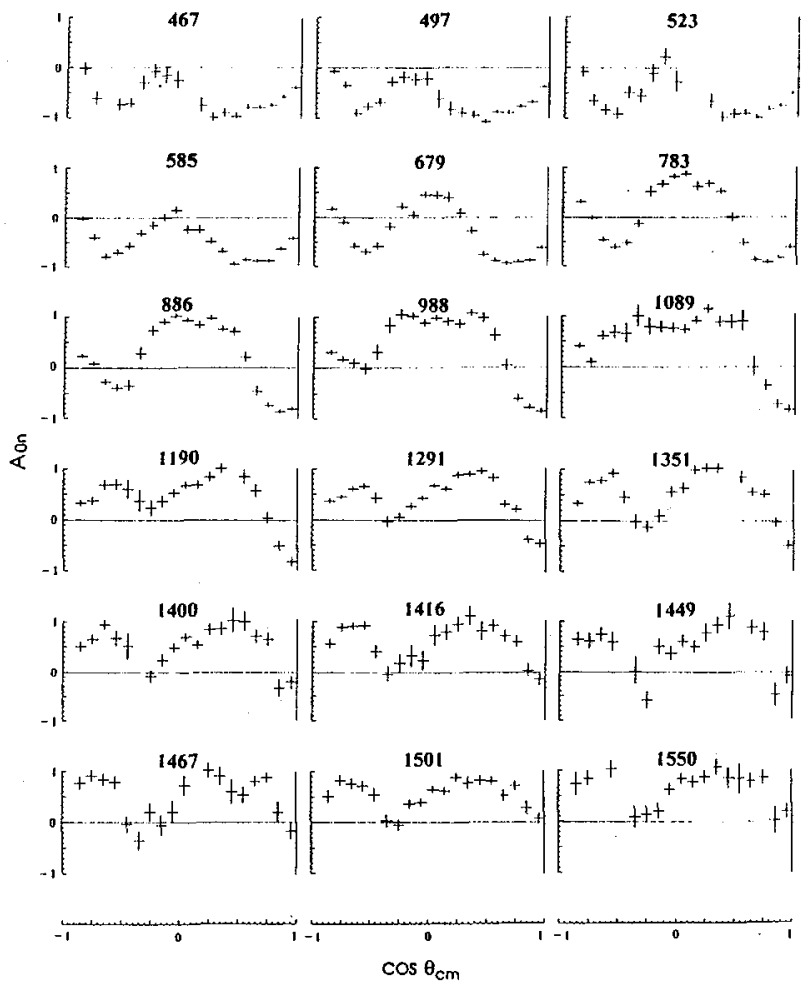

Figure 9: Analysing power for $\overline{\mathrm{p}} \mathrm{p} \rightarrow \pi^{-} \pi^{+}$at different $\overline{\mathrm{p}}$ momenta, as measured by experiment PS172.

Figure 10: Analysing power for $\overline{\mathrm{p}} \mathrm{p} \rightarrow \overline{\mathrm{in}}$ at $656 \mathrm{MeV} / \mathrm{c}$, as measured by experiment PS199, compared with potential model calculations.

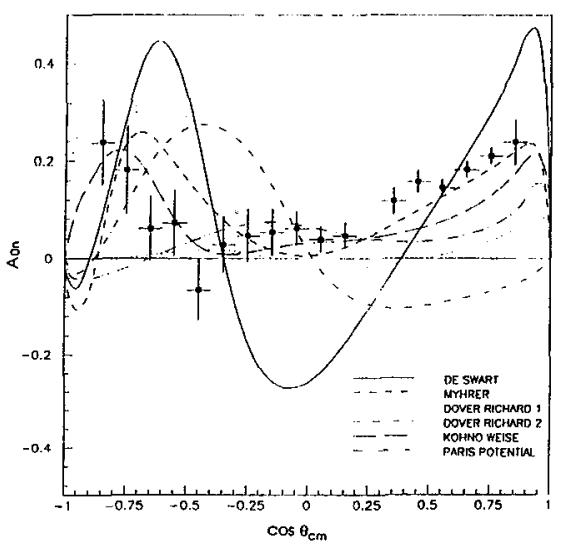


$\mathrm{MeV} / \mathrm{c}$ are shown in Fig. 10, and compared with potential model calculations $[24],[25],[27],[34],[35]$.

The data exhibit an interesting pattern, with backward and forward peaks of similar strengths, and possibly a flat minimum in the central region. This simple pattern is reproduced only poorly by the theoretical predictions, apart from the prediction of Ref. [35]. In particular, there are very big differences in the predictions of the various models also in the forward angles, where $\pi$ exchange is expected to dominate the reaction.

\section{THEORETICAL UNDERSTANDING}

While the cross-section data can be explained also by very 'simple' models, at present the set of $A_{0 n}$ data of $\overline{\mathrm{p}} \mathrm{p} \rightarrow \overline{\mathrm{p}} \mathrm{p}$ and the $A_{0 n}$ measurement of $\overline{\mathrm{p}} \mathrm{p} \rightarrow \overline{\mathrm{n}} \mathrm{n}$ at $656 \mathrm{MeV} / \mathrm{c}$ are not reproduced by any of the existing models. In particular, it does not seem possible to reproduce any of the $A_{0 n}$ data with an OBE potential model in which only a $\pi$ is exchanged [36].

The availability of the new LEAR data has stimulated theoretical work on the models, where, so far, several parameters were only loosely constrained. This is the case, for instance, for the Paris model [37], where a slight readjustment of the core parameters of both the real and the imaginary parts of the potential could improve the calculation of both the charge-exchange cross-section and the analysing power of the elastic channel (Figs. 11a-d).

This is true also in the case of the Dover-Richard model. In order to enhance the spin effects, namely the difference in the strength of the singlet and the triplet part of the meson-exchange potential, they reduced [36] somewhat $r_{\text {cut }}$, the radius at which they regularize the Yukawa potentials. Changing $r_{\text {cut }}$ from 0.8 to $0.74 \mathrm{fm}$, they could obtain a substantial improvement in their calculation

Figure 11: Comparison between data and calculations with the original Paris model (dotted curves) and after adjustment of the core parameters (full curves). Charge-exchange differential cross-section data at 550 $\mathrm{MeV} / c$ (a) from Ref. [18] and at $687 \mathrm{MeV} / c$ (b) from Ref. [20]. Elastic $\bar{p} p$ scattering analysing power data at $697 \mathrm{MeV} / \mathrm{c}$ (c) from Ref. [12] and at $783 \mathrm{MeV} / c$
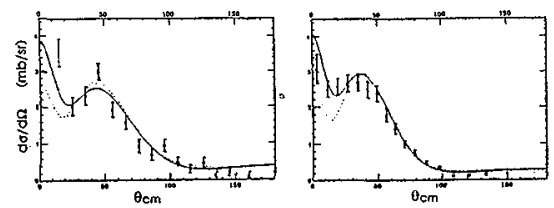

(d) from Ref. [11]

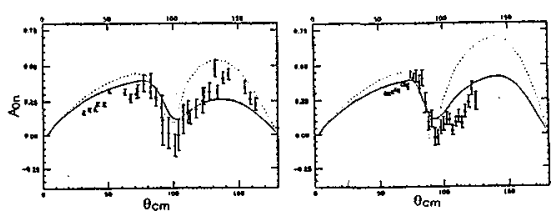




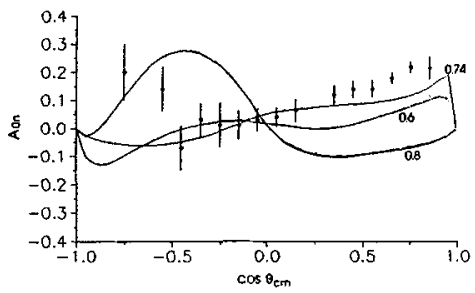

(a)

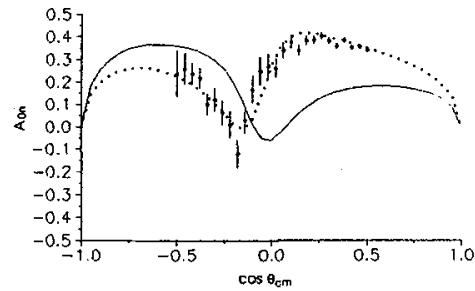

(b)

Figure 12: Comparison of modified Dover-Richard model with $A_{0 n}$ data from Experiments PS199 and PS172. (a): $\bar{p} p \rightarrow$ nin data at $656 \mathrm{MeV} / c$ compared with model calculations with $r_{c u t}=0.8,0.6$, and $0.74 \mathrm{fm} ;(b): \bar{p} p \rightarrow \bar{p} p$ data at $679 \mathrm{MeV} / \mathrm{c}$ with the modified Dover-Richard model (dotted curve) and with a calculation using $\pi$-exchange only (full curve).

of $A_{0 n}$ for charge exchange (Fig. 12a) and at the same time a good agreement for the elastic channel (Fig. 12b).

Improving the models by taking into account the new data is surely a very important and necessary programme. Still, as stated in Section 2, to my mind the main motivation for studying $N \bar{N}$ scattering is

- to extract the global properties of annihilation;

- to learn about the s-channel effects.

In this respect, the amount of theoretical work to be done is still considerable. A very interesting attempt to evaluate the annihilation potential in a consistent way, is a contribution from the Bonn group to this conference [38]. As illustrated in Fig. 13, they use a 'crude' two-meson annihilation model, and compute the
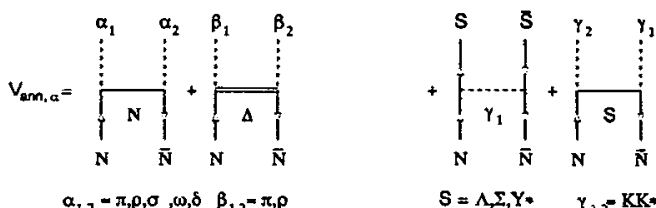

$S=\Lambda, \Sigma, Y * \quad \gamma_{1,2}=\mathrm{KK} *$

Figure 13: Annihilation diagrams included in model B (I) and in model C (I+ II). 
Figure 14: Predictions for the $\overline{\mathrm{p}} \mathrm{p}$ analysing power from the Bonn group. The models A, B, and C are described in the text. Experimental data are from PS172 (open circles) and from PS198 (open triangles).

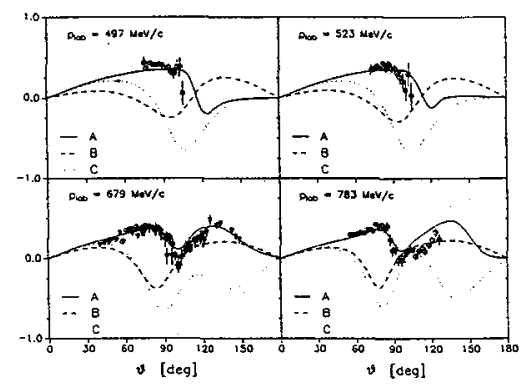

annihilation diagrams by iterating a second time the annihilation interactions $V_{\mathrm{ann}, \alpha}$ which couple to two-meson channels. All meson-baryon vertices have been chosen in consistency with the Bonn potential, which they use to compute the boson-exchange part of the potential. In model B they introduced only nucleon- and $\Delta$-exchange diagrams, while in model $C$ strange hadrons were also considered: model $\mathrm{A}$ is a purely phenomenological complex optical potential (state and energy independent).

Figure 14 compares the results of the calculations of the three models with some $\bar{p} p$ elastic data from experiments PS172 and PS198. While the simple parametrization A gives a surprisingly good description, the much more refined models $\mathrm{B}$ and $\mathrm{C}$ fail to reproduce the experimental data, indicating that more diagrams have to be taken into account.

For the reasons $I$ gave in subsection 3.3, I expect that much insight could be gained if one understood the charge-exchange $A_{0 n}$ data of Fig. 10. Although in the forward direction $\pi$-exchange gives the leading contribution to the crosssection, it does not contribute to $A_{0 n}$ to first order, in Born approximation, and this could explain the broad range of variation in the model calculations. Polarization is contributed by an $\bar{L} \cdot \bar{S}$ term, which could be generated by a $\pi$-exchange only by iteration. Alternatively, spin-orbit term can be generated to first order by a vector exchange (i.e. $\rho$ ). Also, the annihilation potential could contain an $\bar{L} \cdot \bar{S}$ term. So far it does not seem easy to draw a definite conclusion, but this should be possible when the complete set of $A_{0 n}$ data, at all momenta, is available. 


\section{FUTURE PROGRAMMES}

In spite of the good systematic work carried out in $\bar{p} p$ elastic and $\bar{p} p \rightarrow \bar{n} n$ charge exchange, where essentially complete sets of measurements of $\mathrm{d} \sigma / \mathrm{d} \Omega$ and $A_{0 n}$ exist down to $180 \mathrm{MeV} / \mathrm{c}$ and $500 \mathrm{MeV} / \mathrm{c}$ respectively, two-spin observables are needed to constrain the models. I do not think systematic programme of measurements, similar to the one performed on the NN channel, should be started: a phase-shift analysis in the $N \bar{N}$ system would require 20 parameters per partial wave, so it is absolutely ruled out. Also, the dibaryon experience would surely stop, from the beginning, any attempt to go in that direction. On the contrary, in the $N \bar{N}$ case I believe that, after adjustment to the existing data, the potential models should be tested against a few specific measurements, which can select some basic properties of the theory.

Ideally this programme requires polarized $\overline{\mathrm{p}}$ 's on a polarized target. Twospin correlation measurements would then also be possible in some very important annihilation channels, such as $\overline{\mathrm{p}} \mathrm{p} \rightarrow \pi^{+} \pi^{-}, \mathrm{K}^{+} \mathrm{K}^{-}$. Work is going on in the direction of polarizing the LEAR beam: this is what I call the far future. On the other hand, some measurements can be performed already in the next three years or so, using unpolarized $\bar{p}$ 's, polarized proton and neutron targets, and analysing the polarization of one of the particles in the final state; I will start with this topic.

\subsection{Near-future programmes}

Experiment PS199 is the only experiment presently installed at LEAR working on $\mathrm{N} \overline{\mathrm{N}}$ scattering (not to mention the already-quoted experiment PS185 [2], dedicated to $\bar{p} \mathrm{p} \rightarrow \overline{\mathrm{Y}} \mathrm{Y}$ ). In the second half of this year, it is scheduled to measure $D_{0 n 0 n}$ at two momenta ( 500 and $1000 \mathrm{MeV} / \mathrm{c}$ ), by measuring the neutron polarization in $\bar{p} p \rightarrow \vec{n} n$ : at the same time data for $K_{n 00 n}$ will be collected by analysing the $\overline{\mathrm{n}}$ polarization by $\overline{\mathrm{n}} \mathrm{p}$ scattering. The $\overline{\mathrm{n}} \mathrm{p}$ analysing power is not known yet, but the same Collaboration plans to measure it in the future.

The Collaboration has proposed [39] to extend the present program and perform four measurements, some of which have also technical implications:

a. Measurement of $A_{0 n}$ for $\overline{\mathrm{p}} \mathrm{n} \rightarrow \overline{\mathrm{p}} \mathrm{n}$;

b. Measurement of $K_{k 00 k}$ for $\overline{\mathrm{p}} \mathrm{p} \rightarrow \overline{\mathrm{n}} \mathrm{n}$;

c. Measurement of $D_{0 n 0 n}$ for $\overline{\mathrm{p} p} \rightarrow \overline{\mathrm{p}} \mathrm{p}$;

d. Measurement of $\Delta \sigma_{\mathrm{T}}$ for $\overline{\mathrm{p}} \mathrm{p}$. 
Each of these measurements should typically be carried out at three momenta. I will only comment on the second measurement, which is a test of the prediction, put forward ten years ago [40], that the $\overline{\mathrm{n}}$ produced in the forward direction in a charge-exchange reaction on longitudinally polarized protons should be almost fully polarized ( $K_{k 00 k}$ should be close to 1 ). This prediction is expected to be very firm, being based on the tensor character of the long-range $\pi$-exchange. If verified, it suggests a way to produce polarized $\overline{\mathbf{n}}$ beam.

\subsection{Far-future programmes}

A major advance for all future programmes would be achieved if the $\bar{p}$ beam circulating in LEAR could be polarized.

Steffens has described in his talk [41] the project that the FILTEX Collaboration is carrying on, to develop and install in LEAR a gas target of polarized hydrogen [42]. The target would act as a spin filter, by absorbing in a different way the beam component with spin parallel or antiparallel to the target. Also, the gas target will allow one-spin measurements $\left(A_{0 n}\right)$ in $\bar{p}$ p elastic, chargeexchange, and possibly in some annihilation channels at very low momenta. For the circulating $\bar{p}$ beam to be polarized, three conditions must be verified: a minimum density for the target $\left(\sim 10^{14}\right.$ atoms per $\left.\mathrm{cm}^{2}\right)$ thus requiring the use of a storage cell, a minimum difference in the absorption cross-section for parallel and antiparallel spins $\left(\Delta \sigma_{\mathrm{T}} / \sigma_{\mathrm{T}} \sim 0.10\right)$, and the absence of depolarizing effects over the time $(10 \mathrm{~h})$ the beam polarization builds up. A test experiment with protons is at present installed at the Test Storage Ring in Heidelberg.

An alternative way to polarize the circulating $\bar{p}$ beam in LEAR has been proposed [43], which relies on the possibility of spatially separating the two spin components of the beam by means of repeated Stern-Gerlach kicks provided by the so-called spin-splitter, an arrangement of two quadrupoles with a solenoid in between, inserted in a straight section of LEAR. The feasibility of the method is not clear, since the effect is very small (particles with opposite spin directions are separated at a speed of $\sim 2 \mathrm{~mm} / \mathrm{h}$ ) and the integration of the spin-splitter in the lattice of LEAR is not straightforward. A test experiment has been approved at the Indiana cooler ring, and a feasibility test is going on at present $t^{5}$.

\section{CONCLUSIONS}

With the advent of LEAR it is now possible to investigate the $N \bar{N}$ system at low energy, with acceptably precise experiments, and gain new insight into the nuclear force. Although the precision of the experiments is substantially smaller than in the NN case, still it is adequate to extract from the interaction the part which is specific to the $N \vec{N}$ system, namely annihilation.

\footnotetext{
${ }^{5}$ Experiment CE/ 6 at the Indiana University Cyclotron Facility, spokesman A. Penzo.
} 
In the $\overline{\mathrm{p} p} \rightarrow \overline{\mathrm{p}} \mathrm{p}$ elastic, the $\overline{\mathrm{p}} \mathrm{p} \rightarrow \overline{\mathrm{n}}$ charge-exchange, and in the twomeson annihilation $\overline{\mathrm{p} p} \rightarrow \pi^{+} \pi^{-}$and $\overline{\mathrm{p}} \mathrm{p} \rightarrow \mathrm{K}^{+} \mathrm{K}^{-}$channels, complete sets of differential cross-section and analysing power data exist down to 200 and 500 $\mathrm{MeV} / \mathrm{c}$ respectively. These data are only poorly reproduced by the existing $\mathrm{OBE}$ potential models, whose parameters were adjusted essentially for cross-section data before the 1980's. Theoretical work has now started, and it will greatly benefit from the two-spin measurements which are planned over the next few years.

In the future the main goal will be to dispose of polarized $\bar{p}$ and $\bar{n}$ beams. In the case of $\bar{p}$ 's, two different techniques seem promising, and are now under test at proton storage rings. By the time of our next conference, polarization might really be a sophisticated option for the LEAR beams, and an essential ingredient of $N \bar{N}$ physics.

\section{References}

[1] Lefèvre P., LEAR, present status, future and developments, in Physics at LEAR with Low-Energy Antiprotons, Proc. LEAR Workshop, Villarssur-Ollon, 1987 (to be referred to here as Villars 87), eds. C. Amsler, G. Backenstoss, R. Klapisch, C. Leluc, D. Simon, and L. Tauscher (Harwood Academic Publishers), p. 19.

[2] Barnes, P.D. et al., Investigation of the reaction $\mathrm{p} p \rightarrow \bar{\Lambda} \Lambda$ close to threshold at LEAR, contribution $18 \mathrm{D}$ to this conference.

[3] Bryan, R.A., and R.J.N. Phillips, Nuclear Phys. B5 (1968) 201.

[4] Clough, A., et al., Phys. Lett. B146 (1984) 299. Bugg, D.V., et al., Phys. Lett. B194 (1987) 563.

[5] Brückner, W., et al., Z. Phys. A335 (1990) 217.

[6] Kamae, T., et al., Phys Rev. Lett. 44 (1980) 1439.

Sumiyoshi, T., et al., Phys. Rev. Lett. 49 (1982) 628.

[7] Hamilton, R.P., et al., Phys. Rev. Lett. 44 (1980) 1182.

[8] See, for instance, Montanet, L., G.C. Rossi and G. Veneziano, Phys. Rep. C63 (1980) 149.

[9] Armstrong, T., et al., Phys. Rev. D36 (1987) 659.

[10] Brückner, W., et al., Phys. Lett. B116 (1986) 113.

[11] Kunne, R., et al., Phys. Lett. B206 (1988) 557 and Nucl. Phys. B323 (1989) 1 . 
[12] Bertini, R., et al., Phys. Lett. B228 (1989) 531.

[13] Kageyama, T., et al., Phys. Rev. D35 (1987) 2655.

[14] Eisenhandler, E., et al., Nucl. Phys. B113 (1976) 1.

[15] Brückner, W., et al., Phys. Lett. B158 (1985) 180 and in Villars 87, p. 277.

[16] Linssen, L., et al., Nucl. Phys. A469 (1987) 726.

Schiavon, P., et al., Nucl. Phys. A505 (1989) 595.

[17] Kroll, P., and W. Schweiger, Analysis of low-energy antiproton-proton forward scattering, Wuppertal Int. Rep., WUB 89-1 (1989).

[18] Brückner, W., et al., Phys. Lett. B169 (1986) 302.

[19] Bogdanski, M., et al., Phys. Lett. B62 (1976) 117.

[20] Nakamura, K., et al., Phys. Rev. Lett. 53 (1984) 885.

[21] Birsa, R., et al., Measurement of the analysing power and the differential cross-section of the p p charge-exchange reaction at LEAR, CERN-EP/9068 (1990), accepted for publication in Phys. Lett. B, and contribution 4A to this conference.

[22] See, for instance, W. Weise, Low energy antinucleon-nucleon potentials, in Villars 87, p. 287.

[23] Shibata, T., Phys. Lett. $\mathbf{B 1 8 9}$ (1987) 232.

[24] Dover, C. and J.M. Richard, Phys. Rev. C21 (1980) 1466.

[25] Cote, J., et al., Phys. Rev. Lett. 48 (1982) 1319.

[26] Perrot-Kunne, F., Measurement of the analysing power in pp scattering, contributed talk to the First Biennial Conference on Low Energy Antiproton Physics, Stockholm, July 1990 (to be referred to here as LEAP 90).

[27] Timmermans, R.G.E., Th.A. Rijken and J.J. de Swart, Preliminary results obtained with the Nijmegen P-matrix model, private communication from J.J. de Swart.

[28] Albrow, M.G., et al., Nucl. Phys. B37 (1972) 349.

[29] Kunne, R., First measurement of $D_{n n}$ in $\bar{p}$ p elastic scattering, PS172, contributed talk to LEAP 90. 
[30] Birsa, R., et al., Asymmetry measurements in antiproton-proton annihilation into two mesons at low energies, Proc. 9th European Symposium on Proton-Antiproton Interactions and Fundamental Symmetries, Mainz, 1988, eds. K. Kleinknecht and E. Klempt, in Nucl. Phys. B (Proc. Suppl.) 8 (1989) 141.

Tessarotto, F., Spin effects in $\overline{\mathrm{p}} \mathrm{p} \rightarrow \pi^{+} \pi^{-}$and $\overline{\mathrm{p}} \mathrm{p} \rightarrow \mathrm{K}^{+} \mathrm{K}^{-}$at LEAR, PS172, contributed talk to LEAP 90.

[31] Moussallam, B., Nucl. Phys. A429 (1984) 429, and private communication.

[32] Martin, A.D., and M.R. Pennington, Nucl. Phys. B169 (1980) 216.

Martin, B.R., and D. Morgan, Nucl. Phys. B176 (1980) 355.

[33] Le Du, P., et al., Phys. Lett. B44 (1973) 390.

[34] Dalkarov, O.D. and F. Myhrer, Nuovo Cimento A40 (1977) 152.

[35] Kohno; M., and W. Weise, Nucl. Phys. A454 (1986) 429.

[36] Private communication from G. Ihle and J.M. Richard.

[37] Pignone, M., et al., Recent antiproton-proton data and the Paris N $\bar{N}$ potential, contribution to LEAP 90.

[38] Haidenbauer, J., et al., $N \bar{N}$ analysing powers predicted by different annihilation models, contribution $13 \mathrm{~A}$ to this conference.

[39] Macciotta, M.P., et al., Extension of experiment PS199: further study of the spin structure of $\bar{p} \mathrm{~N}$ scattering at LEAR, proposal CERN-PSCC/9016, PSCC/P93 Add. 2, 4 July 1990.

[40] Richard, J.M., Proc. Int. Symposium on High Energy Physics with Polarized Beams and Polarized Targets, Lausanne, 1980, eds. by C. Joseph and J. Soffer (Birkhäuser, Basle, 1980), p. 535.

[41] Steffens, E., Experiments with dense polarized internal targets, invited talk at this conference.

[42] Dobbeling, $\mathrm{H}$., et al., Measurement of spin-dependence in $\bar{p}$ p interaction at low momenta, proposal CERN-PSCC/85-80, PSCC/P92, 5 Nov. 1985.

[43] Akchurin, N., et al., The spin splitter: study of a method for polarizing antiprotons at LEAR, Proposal CERN-PSCC/89-22, PSCC/P120, 23 May 1990. 